\title{
Locomotion of a bioinspired flyer powered by one pair of pitching foils
}

\author{
Xiang Zhang, Guowei He, Shizhao Wang, and Xing Zhang* \\ The State Key Laboratory of Nonlinear Mechanics, Institute of Mechanics, Chinese Academy of Sciences, \\ Beijing 100190, China \\ and School of Engineering Science, University of Chinese Academy of Sciences, Beijing 100049, China
}

(Received 18 July 2017; published 24 January 2018)

\begin{abstract}
We numerically investigate the flight dynamics and aerodynamics of a two-dimensional model for the jellyfishlike ornithopter recently devised by Ristroph and Childress [L. Ristroph and S. Childress, J. R. Soc. Interface 11, 20130992 (2014)]. This simplified model is composed of two rigid thin foils which are forced to pitch in antiphase fashion. The Navier-Stokes equations for the fluid and the dynamics equations for the flyer are solved together in the simulations. We first consider the constrained-flying condition where the flyer model is only allowed to move in the vertical direction. The influences of the control parameters on the hovering performance are studied. With the variations in parameter values, three different locomotion states, i.e., ascending, descending, and approximate hovering, are identified. The wake structures corresponding to these three locomotion states are explored. It is found that the approximate hovering state cannot persist due to the occurrence of wake symmetry breaking after long-time simulation. We then consider the free-flying condition where the motions in three degrees of freedom are allowed. We study the postural stability of a flyer, with its center of gravity located at the geometric center. The responses of the flyer at different locomotion states to physical and numerical perturbations are examined. Our results show that the ascending state is recoverable after the perturbation. The descending state is irrecoverable after the perturbation and a mixed fluttering and tumbling motion which resembles that of a falling card emerges. The approximate hovering state is also irrecoverable and it eventually transits to the ascending state after the perturbation. The research sheds light on the lift-producing mechanism and stability of the flyer and the results are helpful in guiding the design and optimization of the jellyfishlike flying machine.
\end{abstract}

DOI: 10.1103/PhysRevFluids.3.013102

\section{INTRODUCTION}

Flying and swimming animals are the sources of inspiration for devising biomimetic aerial and underwater vehicles. Understanding the aerodynamics (or hydrodynamics) of animal locomotion in air (or water) is essential for optimizing the biomimetic designs. To build an artificial flyer which is capable of hovering, it is natural to mimic the insects which are able to generate sufficient lift without a forward speed. Several different strategies have been observed in the hovering flights of insects [1]: the lift-based stroke in a horizontal plane (also termed the normal mode), the drag-based paddling stroke in a vertical plane, and the hybrid lift- and drag-based stroke in a tilted plane. Three mechanisms were found to be responsible for the generation of large lift: leading-edge vortex [2], wake capture [3], and rapid acceleration and fast pitching up [4]. For hovering insects operating at ultralow Reynolds numbers, the clap-and-fling (interaction between two wings) mechanism can also be used to supplement the lift production [5]. Although the aerodynamics of insects' hovering flights

\footnotetext{
*Corresponding author: zhangx@lnm.imech.ac.cn
} 
(a)

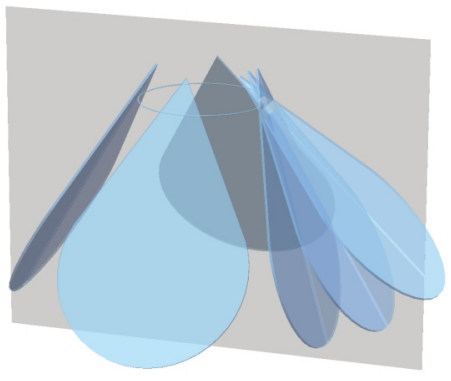

(b)

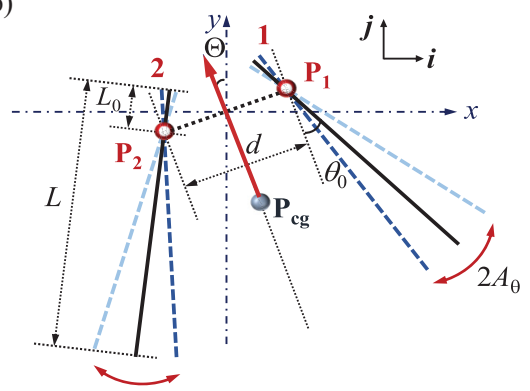

FIG. 1. Schematic diagram (a) for the jellyfishlike ornithopter and (b) the simplified two-dimensional model. Here $P_{1}$ and $P_{2}$ denote the pivot points of the two foils; $P_{c g}$ denotes the center of gravity of the flyer; $\Theta$ is the orientation angle of the flyer; $L, L_{0}$, and $d$ denote the chord length, the distance from the pivot point to the leading end, and the distance between the two pivot points, respectively; $\theta_{0}, \theta(t)$, and $A_{\theta}$ denote the equilibrium angle, the instantaneous pitching angle, and the flapping amplitude, respectively; and $\boldsymbol{i}$ and $\boldsymbol{j}$ denote the unit vectors pointing in the $x^{+}$and $y^{+}$directions, respectively.

are now well understood, due to the inherent instability in the insect-based flapping-wing micro air vehicles (MAVs), complex control systems are needed in the designs. Perhaps for this reason, only a few MAVs capable of untethered hovering flight have been reported [6-12].

Recently, a jellyfishlike ornithopter which can achieve self-righting flight by opening and closing its four wings [see Fig. 1(a)] has been reported [13]. It was shown that the robotic flyer can maintain a stable body orientation during ascending, forward, and hovering flights. To some extent, this robotic flyer shared some similarities with the rigid or flexible objects which were able to hover in oscillating flows [14-18]. Although the promise of this design strategy has been demonstrated in test-flying the prototype, the underlying physical mechanisms which govern its locomotion capabilities are still not well understood.

Despite the term "jellyfishlike" [sic] used in [13] to describe the flying machine, there are some apparent differences between the kinematics of its flapping wings and that of a jellyfish's bell. Jellyfish uses very complicated contract and extension of the soft deformable bell during swimming, with some asymmetries in the contracting and expanding phases [19]. The kinematics employed by the flying machine is actually very similar to the simple pitching motion (i.e., rotation about one pivot point), although moderate wing flexion can be observed.

Motivated by the work in [13] and in light of the observation above, in this paper investigations are conducted on a two-dimensional numerical model consisting of one pair of pitching foils [see Fig. 1(b)]. Although the complicated three-dimensional effects and the wing flexion are excluded in the model, this study can still shed light on the aerodynamic characteristics of the flying machine reported in [13]. It should be noted that some investigations have been conducted before on the dual pitching-foil system under either tethered or free-swimming condition [20,21]; however, the performance of such system in flying against gravity has received little attention.

More recently, a similar two-dimensional model for the jellyfishlike flyer has been studied numerically by using a vortex sheet method [22] and some results regarding the flyer's postural stability were found to be inconsistent with the experimental observations in [13]. Unlike the work in [22], where the flows were in the limit of infinite Reynolds number, in the present work we focus on the regime of intermediate Reynolds numbers by numerically solving the full Navier-Stokes equations. Particular attention is given to the exploration of various wake structures associated with different locomotion states, and the link between wake structure and hovering performance.

The arrangement of the rest of the paper is as follows. The problem description and numerical method are introduced in Sec. II. The simulation results are presented in Sec. III. First, the influences of some important control parameters on the hovering performance are investigated. Next, the 
emergent wake structures under the constrained-flying condition (where only the vertical motion is permitted) are explored. Finally, the postural stability of the flyer under the free-flying condition is studied. In Sec. IV, we summarize the conclusions and also discuss future research avenues.

\section{PROBLEM DESCRIPTION AND NUMERICAL METHOD}

\section{A. Computational model}

In this study, we consider one pair of rigid thin foils which pitch in antiphase fashion [see Fig. 1(b)]. The motions of the foils can be prescribed as

$$
\theta_{1,2}(t)= \pm\left[\theta_{0}+A_{\theta} \cos (2 \pi f t)\right]
$$

where $\theta_{1}$ and $\theta_{2}$ are the instantaneous pitching angles, $\theta_{0}$ is the equilibrium angle, and $f$ and $A_{\theta}$ are the flapping frequency and flapping amplitude, respectively.

The fluid flow is assumed to be two dimensional and laminar. The motion of the fluid is governed by the incompressible Navier-Stokes equations, which can be written in a dimensionless form as

$$
\begin{aligned}
\frac{\partial \boldsymbol{u}}{\partial t}+(\boldsymbol{u} \cdot \nabla) \boldsymbol{u} & =-\nabla p^{\prime}+\frac{1}{\operatorname{Re}} \nabla^{2} \boldsymbol{u}+\boldsymbol{f}, \\
\nabla \cdot \boldsymbol{u} & =0 .
\end{aligned}
$$

Here $\boldsymbol{u}$ is the fluid velocity, $p^{\prime}$ is the summation of the pressure and the gravitational potential, and $f$ is the momentum source term, which represents the force exerted by the immersed foils. The reference length, time, and velocity used in the nondimensionalization are $L, 1 / f$, and $L f$, respectively. The Reynolds number in Eq. (2a) is defined as $\operatorname{Re}=L^{2} f / v$, where $v$ is the kinematic viscosity of the fluid. Due to the absorption of the gravitational potential into the pressure term, the gravitational force does not appear explicitly in Eq. (2a).

The dimensionless dynamics equations which govern the locomotion of the flyer are

$$
\begin{gathered}
\beta \frac{d^{2} \boldsymbol{X}_{c g}}{d t^{2}}=\sum_{i} \boldsymbol{F}\left(\boldsymbol{X}_{i}\right) \Delta s-\frac{\beta}{\mathrm{Fr}^{2}} \boldsymbol{j}, \\
\frac{d}{d t}\left(I \frac{d \Theta}{d t}\right)=\sum_{i}\left(\boldsymbol{X}_{i}-\boldsymbol{X}_{c g}\right) \times \boldsymbol{F}\left(\boldsymbol{X}_{i}\right) \cdot \boldsymbol{k} \Delta s,
\end{gathered}
$$

where $\boldsymbol{X}_{c g}$ and $\Theta$ are the position vector of the center of gravity and the orientation angle, respectively, $\boldsymbol{F}$ is the distributed Lagrangian force on the flapping foils as a result of the fluid-structure interaction, $\Delta s$ is the width of the Lagrangian grid, $\boldsymbol{k}$ is the unit normal vector which points outward from the $x-y$ plane, and $\beta=\left(\rho_{s} \delta\right) /\left(\rho_{f} L\right)$ and $I=\beta \sum\left(\boldsymbol{X}_{i}-\boldsymbol{X}_{c g}\right) \cdot\left(\boldsymbol{X}_{i}-\boldsymbol{X}_{c g}\right) \Delta s$ are the mass ratio and dimensionless moment of inertia, where $\rho_{s}, \rho_{f}$, and $\delta$ are the density of the foil, the density of the air, and the thickness of the foil, respectively. Here the mass of the load is lumped into the mass of the foils and not treated separately. In addition, $\mathrm{Fr}=f \sqrt{L / g}$ is the Froude number. Note that the gravitational potential absorbed in $p^{\prime}$ can result in a buoyancy force which is proportional to the volume occupied by the foils. Since such volume is sufficiently small, the buoyancy force term is not included in Eq. (3).

In this paper, two types of motion of the flyer, namely, constrained flying and free flying, are considered. For the constrained-flying cases, the flyer is only allowed to move in the vertical direction, while the lateral motion and rotation are prohibited. Under such a condition, only the second component of the position vector in Eq. (3a) needs to be solved. In the situation of free flying, Eqs. (3) need to be solved. 
TABLE I. Values of control parameters used in the simulation. (In searching for cases which precisely satisfy the hovering condition, some additional values not listed in the table are also used in the simulation.)

\begin{tabular}{lc}
\hline \hline Parameters & Values \\
\hline$\beta$ & $0.1,0.2,0.25,0.3,0.4,0.5,0.7,1.0,1.5,2.0$ \\
$d / L$ & $0.5,0.6,0.8,1.0,1.2,1.4,1.6,2.0,2.5,3.0$ \\
$L_{0} / L$ & $0.0,0.1,0.2,0.3,0.4,0.5$ \\
$\theta_{0}$ & $-\pi / 12-\pi / 6\left(\Delta \theta_{0}=\pi / 36\right)$ \\
$A_{\theta}$ & $\pi / 36-\pi / 6\left(\Delta A_{\theta}=\pi / 72\right)$ \\
$\operatorname{Re}$ & $50-1600$ \\
$\mathrm{Fr}$ & $0.5-15$ \\
\hline \hline
\end{tabular}

\section{B. Flow and dynamics solvers}

The Navier-Stokes equations (2) are solved by using the direct-forcing immersed boundary method based on the discrete stream function formulation [23]. The algebraic multigrid method is used to solve the linear systems arising from the discretization. The code is parallelized using the message passing interface protocol [24]. The dynamics equations (3) are solved by using the explicit Eulerian scheme for the temporal discretization. The loosely coupled scheme is used for solving the fluidstructure interaction problem.

Before performing the simulations of this work, the code is first validated by computing the aerodynamic force in the hovering flight of a dragonfly wing model [1,25]. The comparisons of the result with the reference solutions are detailed in Appendix A and the agreements are found to be satisfactory.

\section{SIMULATION RESULTS}

\section{A. Control parameters and numerical settings}

The control parameters used in the simulations are listed in Table I. Some parameters such as the Froude numbers, the density ratio, the flapping amplitude, the equilibrium angle, the pivot point position, and the gap between the two pivot points are chosen to lie in a reasonable range which is comparable to that of the flying machine presented in [13]. The Reynolds numbers in the simulations are one order of magnitude smaller than those in [13]. The reasons for assigning smaller Re in the simulations are twofold. First, due to the mesh resolution requirement for resolving the boundary layer, systematic parametric studies at a higher Re around $10^{4}$ are still prohibitive; second, a saturation behavior in the locomotion performance with increasing Reynolds number has been found in the range of $\operatorname{Re}=10^{2}-10^{3}$ (to be addressed later in Sec. IIIB).

A large rectangular computational domain of $[-12 L, 12 L] \times[-24 L, 24 L]$ is used in the simulations of this study. The no-slip condition on the surface of the flyer is realized by using the immersed boundary technique. It should be noted that the selected thickness value (through the prescribed value of $\beta$ ) only affects the inertial terms in the dynamic equations. In solving the Navier-Stokes equations, the two links of the flyer are treated as very slender bodies with "zero thickness" [23]. The slip-wall boundary condition is imposed on the four outer boundaries of the computational domain. To reduce the total number of grid points while maintaining a sufficiently high resolution near the body, we utilize multiblock Cartesian grids with hanging nodes [23]. The finest grids are deployed in the subdomain of $[-2 L, 2 L] \times[-16 L, 16 L]$ for the constrained-flying simulations, with the widths ranging from $0.01 L$ to $0.02 L$, depending on the Reynolds numbers. For the free-flying simulations, the subdomain with the finest grid is enlarged to $[-8 L, 8 L] \times[-16 L, 16 L]$ to deal with possible larger lateral displacements of the flyer. The resolution of the Lagrangian grid on the dual flapping foils is the same as that of the Eulerian grid. The time steps in the simulations are chosen to be $0.001 T$ for the ascending and descending cases and $0.0005 T$ for the approximate 
hovering cases (the maximum Courant-Friedrichs-Lewy number never exceeds 0.2 for all cases). The initial positions of the two foils are $\theta_{1}(0)=\theta_{0}+A_{\theta}$ and $\theta_{2}(0)=-\theta_{0}-A_{\theta}$, respectively. The initial velocity of the fluid is set to zero. Numerical tests are conducted to ensure that converged solutions are obtained with the refinement of mesh and time-step sizes. The results of these tests are detailed in Appendix B.

A comprehensive parametric study has been conducted under the constrained-flying condition to investigate the influences of control parameters on hovering performance and wake structure. Meanwhile, a limited number of free-flying cases are also simulated to probe the postural stability of the flyer under perturbations.

\section{B. Effects of parameters on hovering performance in constrained flying}

In the constrained-flying cases, the flyer's locomotion states can be divided into three categories, i.e., ascending, descending, and approximate hovering. We use the terminal vertical velocity $U_{T}$, which is defined as

$$
U_{T}=\frac{1}{t_{1}-t_{0}} \int_{t_{0}}^{t_{1}}\left[\dot{\boldsymbol{X}}_{c g} \cdot \boldsymbol{j}\right] d t,
$$

to identify the locomotion state and quantify the performance of the flyer. The integrand limits in Eq. (4) are chosen such that the averaging is performed within a time interval where the velocity is (periodically) steady. In this paper, $t_{0}=10 T$ is used to exclude the influence of the initial condition, where $T$ is the period of the pitching motion. For most of the cases studied here, $t_{1}-t_{0}=10 T$ is used in the averaging. For a few cases where multiperiodic behavior is exhibited in the velocity, the time intervals used in the averaging may reach up to $30 T$.

The terms "ascending" and "descending" are used to describe the locomotion state when $\left|U_{T}\right|>$ 0.1 and $U_{T}$ is positive or negative, respectively. The term "approximate hovering" is used to describe the locomotion state when $\left|U_{T}\right| \leqslant 0.1$. Note that for the approximate hovering cases, the $U_{T}$ defined here only reflects its temporary locomotion state due to the occurrence of wake symmetry breaking after a long-time simulation (to be discussed later in Sec. III C).

To investigate the sensitivity of the performance to the variation of certain parameters, some base cases are first selected. The criteria for selecting the base cases are that (a) the parameter values should lie in a reasonable range and (b) the dynamic states are the ones that we intend to achieve. An explanation of the reasonable range for the parameter values has been given in Sec. III A. An intended dynamic state (i.e., ascending, hovering, or descending) can be achieved by adjusting the parameters associated with the kinematics (such as $\mathrm{Re}, \mathrm{Fr}$, and $A_{\theta}$ ). This is done by following a cut-and-try procedure. After the base case is selected, the sensitivity study is then conducted by allowing one or more parameter values to vary while keeping others fixed.

\section{Effect of Reynolds number}

We first explore the influence of Reynolds number on the locomotion performance. A series of simulations are conducted by varying $\mathrm{Re}$ in the range of 50-600 while keeping other control parameters fixed.

The terminal vertical velocities as a function of $\mathrm{Re}$ for three sets of control parameters corresponding to the ascending, descending, and approximate hovering states are shown in Fig. 2. From this figure it can be seen that as long as Re is sufficiently high (larger than 200), the Reynolds-number effect becomes insignificant. This observation can be used to further justify the assigning of lower Re in the simulations (when comparing with those in the experiments). Here we offer a caveat in interpreting the saturation behavior shown in Fig. 2. This behavior only confirms that the performance of the flyer is no longer sensitive to the variation of viscosity if the viscosity is sufficient small. The saturation behavior cannot be interpreted as the insensitivity of performance with respect to size variation, because size variation will result in the simultaneous variations in $\mathrm{Re}$, Fr, and $\beta$. 


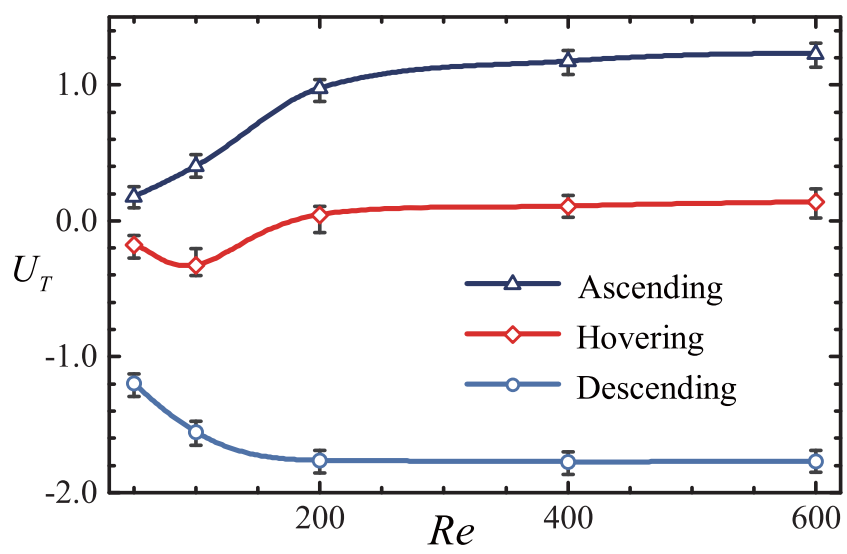

FIG. 2. Terminal vertical velocity as a function of Re. The bars on the curves symbolize the amplitudes of velocity fluctuations. The fixed control parameters are $A_{\theta}=15^{\circ}, \beta=0.5, L_{0}=0.2 L, d=1.0 L$, and $\theta_{0}=0^{\circ}$ for all cases, $\mathrm{Fr}=2.89$ for the ascending cases $(\triangle), \mathrm{Fr}=1.92$ for the approximate hovering cases $(\diamond)$, and $\mathrm{Fr}=0.96$ for the descending cases $(\circ)$.

\section{Effects of flapping frequency and amplitude}

The flapping frequency $f$ and amplitude $A_{\theta}$ are the two most important kinematic parameters which affect the hovering performance. To isolate the effects of varying frequency and amplitude while keeping other parameters unchanged, we construct a two-dimensional parameter space $\left(A_{\theta}, \tilde{f}\right)$ which is spanned by the flapping amplitude $A_{\theta}$ and the normalized frequency $\tilde{f}$. The normalized frequency is defined as $\tilde{f}=f / f_{0}$, where $f_{0}$ is the reference frequency for the case with $\operatorname{Re}=200$ and $\mathrm{Fr}=1.92$. The reason for choosing this reference case is that this is an approximate hovering case for $\beta=0.5$. Since we are particularly interested in the hovering flight of the flyer, by choosing this case as the reference one, we can easily figure out the sensitivity of the hovering performance to the variations of frequency and amplitude. Actually, this choice is somewhat arbitrary and not unique [any case on the black line in Fig. 3(a) can be a good candidate]. Provided that the chord length $L$ is
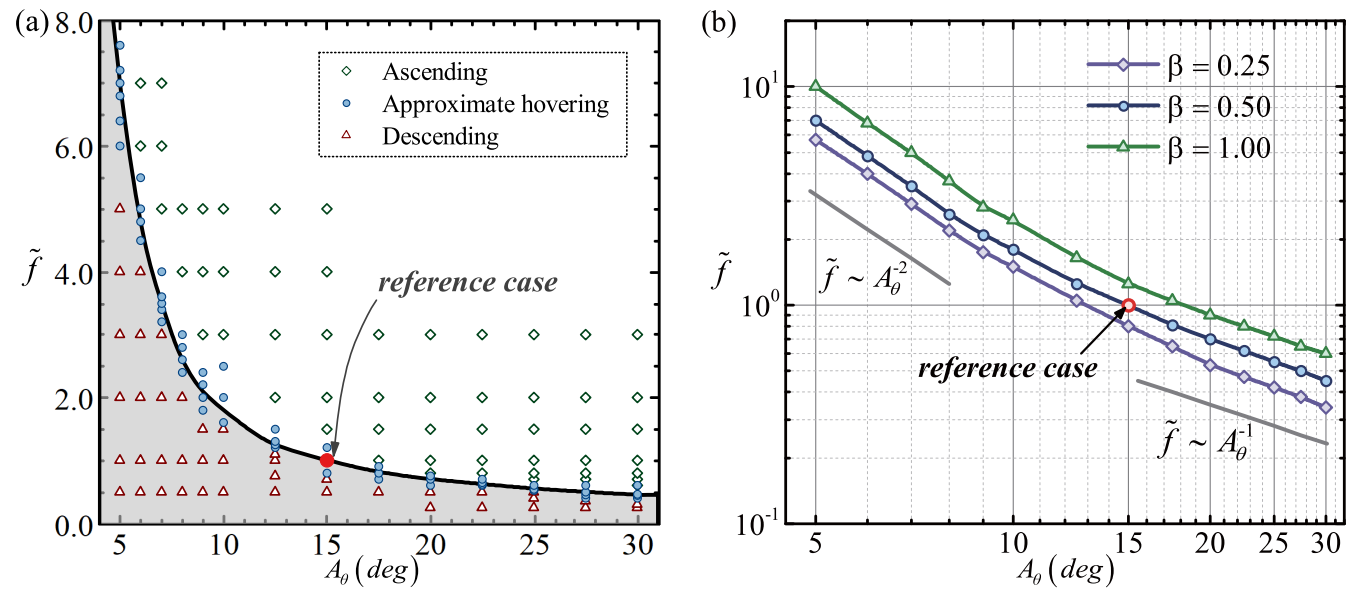

FIG. 3. Influences of flapping frequency and amplitude on the locomotion performance: (a) a phase diagram for the map of locomotion states across $\left(A_{\theta}, \tilde{f}\right)$ at $\beta=0.5$ and (b) the scaling of $\tilde{f}$ with respect to $A_{\theta}$, for cases which approximately satisfy the hovering condition (i.e., cases with the minimal values of $\left|U_{T}\right|$ ) at three different values of $\beta$. The fixed control parameters are $\theta_{0}=0^{\circ}, L_{0}=0.2 L$, and $d=1.0 L$. 
fixed among all cases, $\tilde{f}=\alpha$ thus corresponds to the case with $\operatorname{Re}=200 \alpha$ and $\mathrm{Fr}=1.92 \alpha$, where $\alpha$ is a positive real number.

A phase diagram for the locomotion states across the space of $\left(A_{\theta}, \tilde{f}\right)$ for $\beta=0.5$ is shown in Fig. 3(a). Note that for the cases marked in this figure, all control parameters are fixed, except Re, Fr, and $A_{\theta}$. The ascending cases are found in the upper right region, while the descending cases are found on the lower left. The narrow band which separates these two regions corresponds to the approximate hovering state. The values of $A_{\theta}$ and $\tilde{f}$ which satisfy the hovering condition can be extracted from this figure. Such values extracted at three different values of $\beta(0.25,0.5$, and 1.0) are plotted in Fig. 3(b). It can be seen that $\tilde{f}$ obeys the scaling laws of $\tilde{f} \sim A_{\theta}{ }^{-2}$ and $\tilde{f} \sim A_{\theta}{ }^{-1}$ for $A_{\theta}<10^{\circ}$ and $A_{\theta}>15^{\circ}$, respectively. Thus, the weight-supporting capability of the flyer is proportional to the foil's trailing-end velocity at relatively large flapping amplitudes. At relatively small flapping amplitudes, however, a higher trailing-end velocity is required to support the same weight with decreasing amplitude. It should be noted that the universality of such a scaling law is still not known (it may not be valid if the configuration parameters such as $d / L, L_{0} / L$, and $\theta_{0}$ are different).

\section{Effects of other control parameters}

Next, we study the influences of other control parameters, namely, the density ratio, the pivot point position, the equilibrium angle, and the gap distance between the two foils, on the performance of the flyer. A parametric study is carried out by only varying one parameter at a time while keeping the other parameters fixed. The results of this study are summarized in Fig. 4, which shows the terminal vertical velocity as a function of the varying parameters.

Figure 4(a) shows the terminal vertical velocity as a function of density ratio. It can be seen that the approximate hovering state may transit to an ascending (or a descending) state if the density ratio is reduced (or increased). This is consistent with our intuition, i.e., a lighter flyer tends to rise while a heaver flyer tends to fall. In Fig. 4(b) it is shown that by moving the pivot point towards the leading end (or trailing end), the approximate hovering state may transit to an ascending (or a descending) state. In this scenario, the transition from approximate hovering to ascending (or descending) can be attributed to increased (or decreased) trailing-end velocity. The effect of varying the equilibrium angle on the performance is demonstrated in Fig. 4(c). It can be seen that in the vicinity of the approximate hovering state, the increase of the equilibrium angle can result in the reduction of the terminal vertical velocity. The reversal of this trend near $\theta_{0}=5^{\circ}$ is associated with the sudden change of wake structure: the transformation from one vortex dipole trapped in between two foils into two large vortices outside the two foils (to be addressed later in Sec. IIIC). Figure 4(d) shows the influence of the gap distance between the two pivot points on the terminal vertical velocity. Since the terminal vertical velocity is not very sensitive to the variation of gap distance in the vicinity of the approximate hovering state, here attention is paid to the ascending state only. From Fig. 4(d) it can be clearly seen that the terminal vertical velocity decreases with increased gap distance. The influences of the equilibrium angle and the gap distance on the performance can be interpreted as the enhancement (or reduction) of the mutual interaction between two foils in close proximity (or inviscid ground effect due to symmetry [26]).

\section{Wake structures under the constrained-flying condition}

We now look at the wake structures that emerge under the constrained-flying condition. The typical wake structures corresponding to the three locomotion states are shown in Fig. 5. For the ascending state [Fig. 5(a)], two vortices with opposite signs form a dipole and are shed from the two trailing ends during one flapping cycle. These dipoles induce downward jet flows with the lateral velocity components pointing outward from the centerline. Owing to the steadily ascending motion, the vortices shed in the previous flapping cycles are kept away from the flyer. This wake structure resembles the one produced by a swimming two-dimensional jellyfish model [19]. For the descending state [Fig. 5(b)], the emergent wake structure is composed of two parallel von Kármán vortex streets, 
(a)

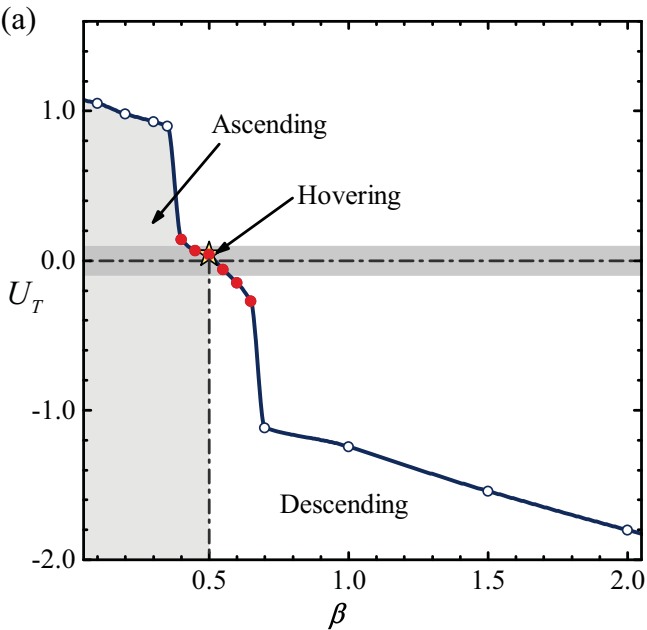

(c)

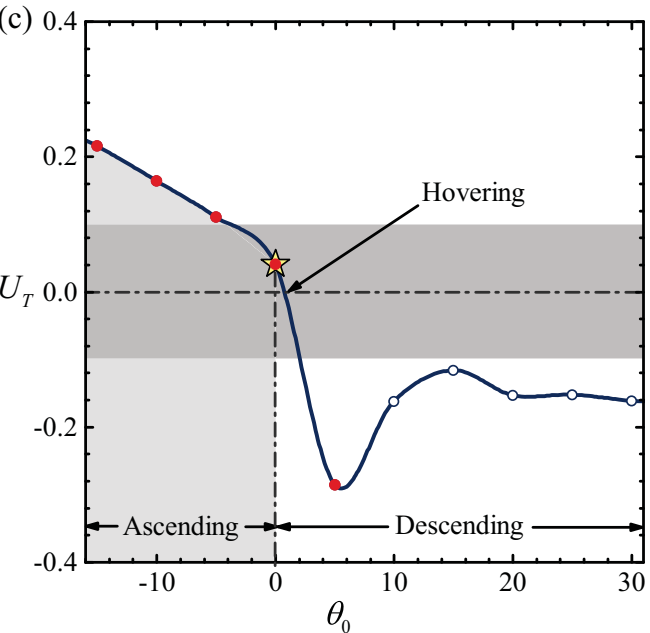

(b)

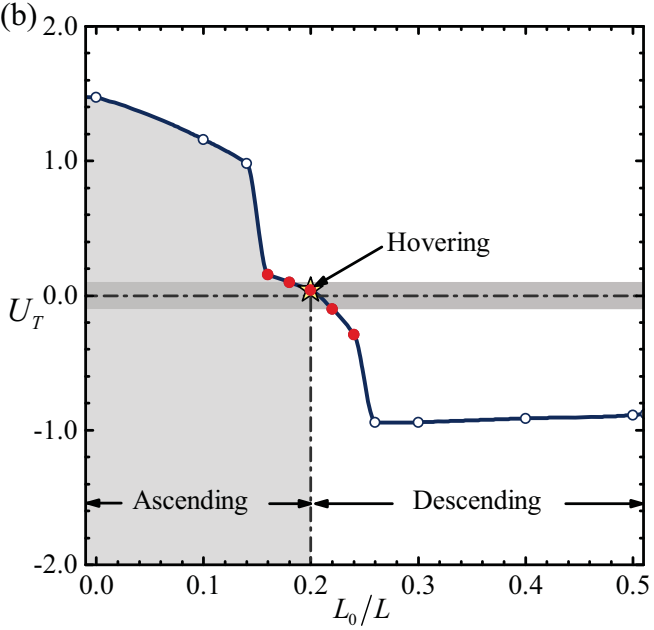

(d)

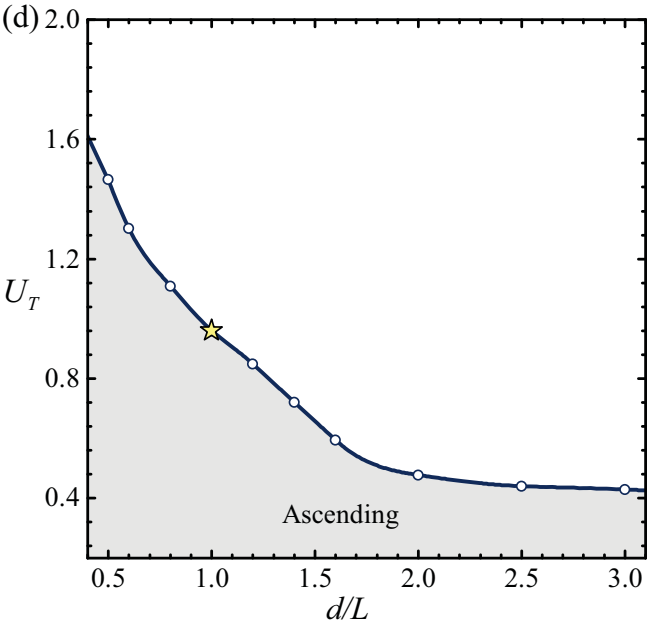

FIG. 4. Terminal vertical velocity as a function of (a) density ratio, (b) pivot point position, (c) equilibrium angle, and (d) gap distance between the two pivot points. The closed and open circles denote the cases with and without the occurrence of wake symmetry breaking, respectively. The control parameters for the cases denoted by a star on the curves in (a)-(c) are $\operatorname{Re}=200, \mathrm{Fr}=1.92, A_{\theta}=15^{\circ}, \beta=0.5, \theta_{0}=0^{\circ}, L_{0}=0.2 L$, and $d=1.0 L$. The control parameters for the case denoted by a star on the curve in (d) are $\operatorname{Re}=300, \mathrm{Fr}=2.89$, $A_{\theta}=15^{\circ}, \beta=0.5, \theta_{0}=0^{\circ}, L_{0}=0.2 L$, and $d=1.0 L$.

which are the typical footprints left by free-falling objects. In the vortex streets, vortices shed in sequential order are arranged in a staggered fashion.

For the approximate hovering state, one salient feature is the lingering of large chunks of vorticity near the body. Due to the lack of a steady translational motion, the vorticity produced by the successive flapping is continuously fed into certain wake structures and also accumulated within these structures. The visual appearance of the wake structure may differ significantly for different equilibrium angles. In the case of $\theta_{0}=0^{\circ}$, a large vortex dipole is trapped in between the two flapping foils [see Fig. 5(c)]. This dipole possesses very strong vorticity intensity and induces an upward velocity. Besides that, a series of weaker vortex dipoles which induce downward velocity are also found below the strongest dipole. Unlike the dipoles in the ascending and descending states, all dipoles are now squeezed near 

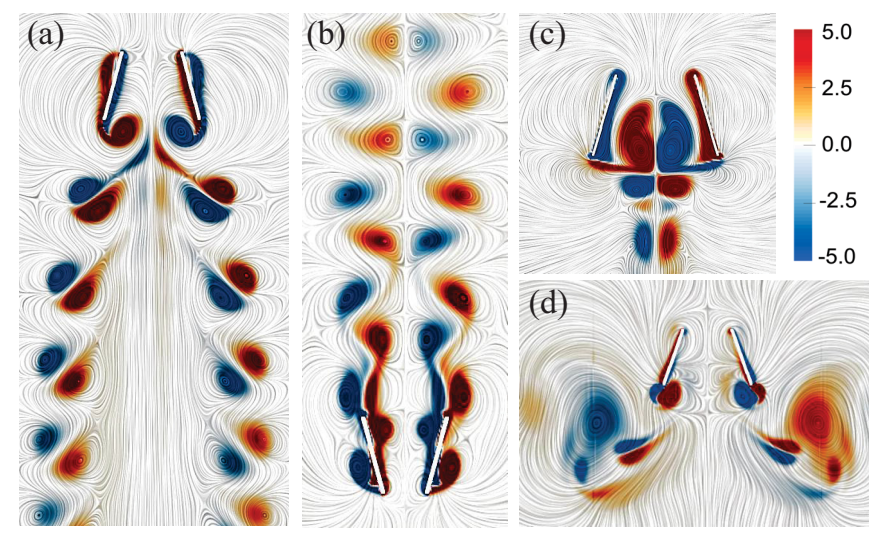

FIG. 5. Wake structures corresponding to different locomotion states: (a) ascending, (b) descending, (c) approximate hovering at $\theta_{0}=0^{\circ}$, and (d) approximate hovering at $\theta_{0}=15^{\circ}$. Vorticity contours and streamlines are plotted for visualizing the vortical structures. The control parameters used in the simulations are $A_{\theta}=15^{\circ}, \beta=0.5, d=1.0 \mathrm{~L}$, and $L_{0}=0.2 \mathrm{~L}$ for all cases and (a) $\operatorname{Re}=300, F r=2.89$, and $\theta_{0}=0^{\circ}$, (b) $\operatorname{Re}=100, \mathrm{Fr}=0.96$, and $\theta_{0}=0^{\circ}$, (c) $\operatorname{Re}=200, \mathrm{Fr}=1.92$, and $\theta_{0}=0^{\circ}$, and (d) $\operatorname{Re}=216, \mathrm{Fr}=2.07$, and $\theta_{0}=15^{\circ}$. Movies S1-S4 which show the animations of vortex structure evolution in the wakes corresponding to (a)-(d) are available as Supplemental Material [27].

the centerline because the lateral components of the dipole-induced velocities are almost zero. In the case of $\theta_{0}=15^{\circ}$, two large vortex cores are found outside the two flapping foils and the far wake is clear of any concentrated vorticity [see Fig. 5(d)]. In the two cases above, the lingering of vortex structures near the flyer can result in strong vortex-body interaction, which in turn has a critical influence on the hovering performance.

Another distinct feature of the approximate hovering state is that its wake is susceptible to the (left-right) symmetry-breaking instability. The occurrence of wake symmetry breaking eventually leads to an irregular (or a chaoticlike) wake pattern as shown in Fig. 6. This instability is an intrinsic property of the system and the symmetry breaking can be triggered by either physical or numerical perturbations. Figure 7 shows the time histories of the vertical velocity and lateral force, with and without the application of a physical perturbation. The physical perturbation considered here is a Gaussian pulse of vertical force (which can be either positive or negative in sign). It can be seen that the ascending and descending states can be recovered some time after the perturbation is applied [see Figs. 7(a) and 7(b)], whereas the approximate hovering state cannot be recovered. For the approximate hovering state, after a long-time simulation the tendency of falling can be clearly seen and large lateral forces are also visible [see Figs. 7(c)-7(f)].
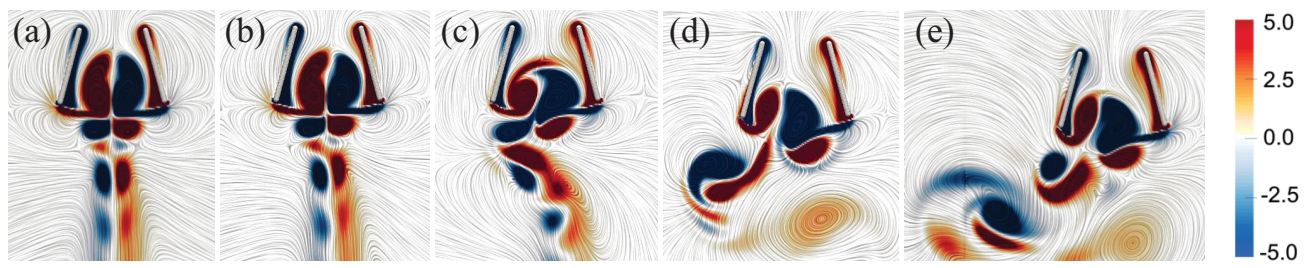

FIG. 6. Asymmetric wake structure corresponding to the approximate hovering state after a long-time simulation at (a) $t=18 T$, (b) $t=20 T$, (c) $t=24 T$, (d) $t=28 T$, and (e) $t=32 T$. The control parameters are the same as those for Fig. 5(c). 

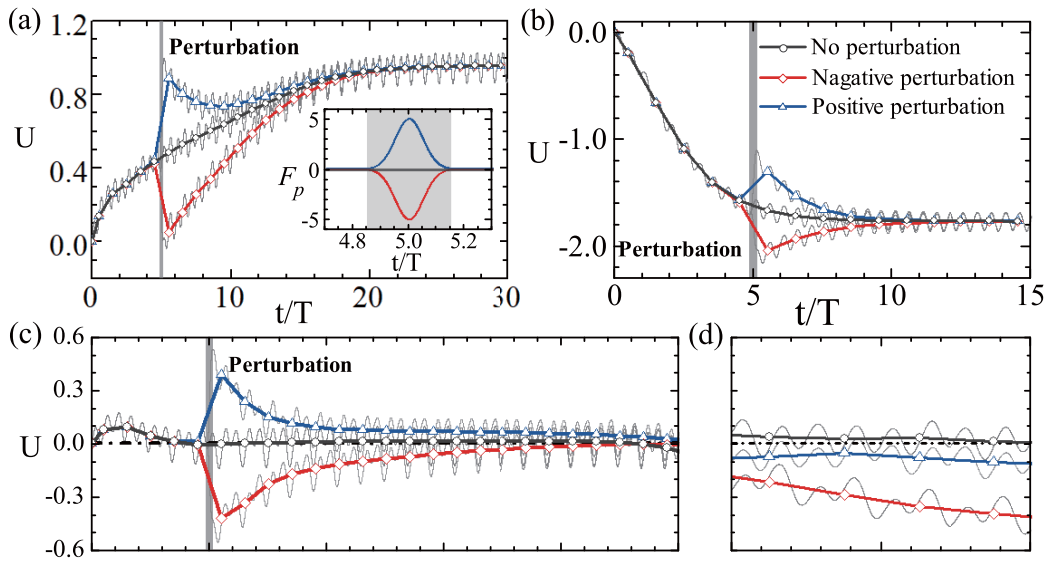

(d)
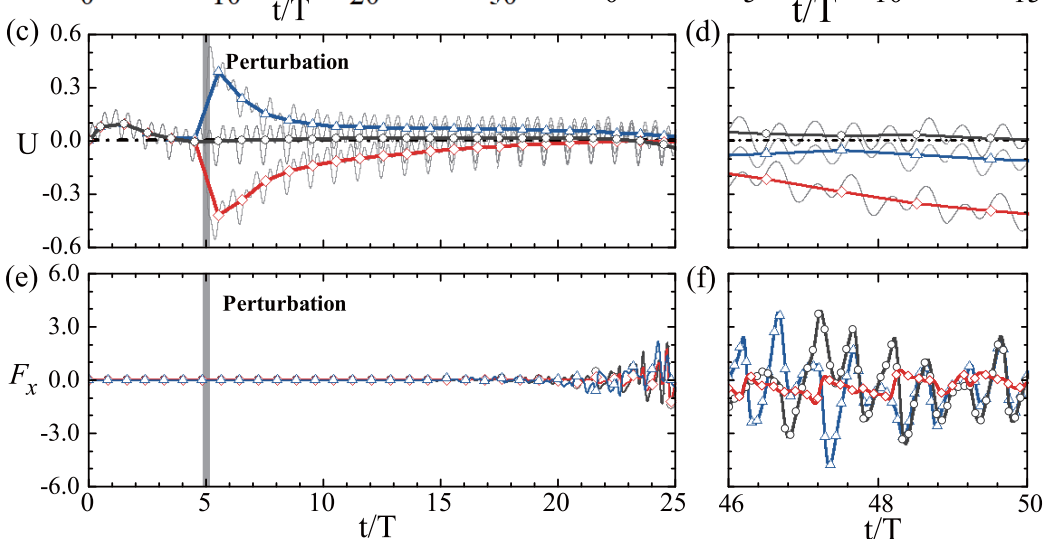

FIG. 7. Time histories of the vertical velocities and lateral forces, with and without the application of physical perturbations: (a) time history of the vertical velocity at the ascending state, (b) time history of the vertical velocity at the descending state, (c) and (d) short- and long-time histories of the vertical velocity at the approximate hovering state, and (e) and (f) short- and long-time histories of the lateral forces at the approximate hovering state. The control parameters used in (a)-(c) are the same as those for Figs. 5(a)-5(c), respectively. The physical perturbations imposed are Gaussian pulses of vertical force in the form of $F_{p}= \pm A_{f} \exp \left[-\left(t-t_{c}\right)^{2} / 2 \tau_{c}{ }^{2}\right]$, which are shown in the inset of (a). The parameters in the Gaussian pulses of vertical force are $A_{f}=5.0$, $t_{c}=5.0$, and $\tau_{c}=0.05$. The thin lines and thick lines in (a)-(d) represent the instantaneous vertical velocities and the average vertical velocities over one flapping cycle, respectively. The thick lines in (e) and (f) represent the instantaneous lateral forces.

From Figs. 7(e) and 7(f) it can also be seen that the wake symmetry breaking eventually occurs after a long-time simulation even without any physical perturbation and the amplitude of the lateral force developed is of the same order as those with the physical perturbations. In this scenario, the only source of perturbation is the round-off error due to machine precision. (To avoid the grid-induced asymmetry, special care is taken in the placement of the two foils on the background Eulerian grid.) Further numerical experiments indicate that after the symmetry breaking is triggered, the evolution of the lateral force becomes very sensitive to the numerical settings such as time step and grid width (results not shown here for brevity). Thus, the so-called mesh-independent and time-step-independent solutions are not achievable for these cases. For the purpose of avoiding uncertainty in evaluating the terminal vertical velocity for the approximate hovering state, the starting and ending time points for performing the averaging in Eq. (4) are selected to ensure that the periodicity in velocity has been fully established already and the wake symmetry breaking has not been triggered yet.

\section{Recoverability of the locomotion states under the free-flying condition}

In this section, the recoverability of the locomotion states under the free-flying condition will be explored. Unlike the constrained-flying cases, now the postural stability becomes a crucial factor in determining the recoverability of the locomotion state. Like that pointed out in [22], the appropriate placement of the center of gravity is essential for maintaining postural stability. The top-heavy 
(a)

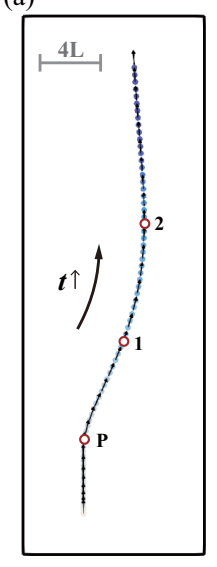

(b)
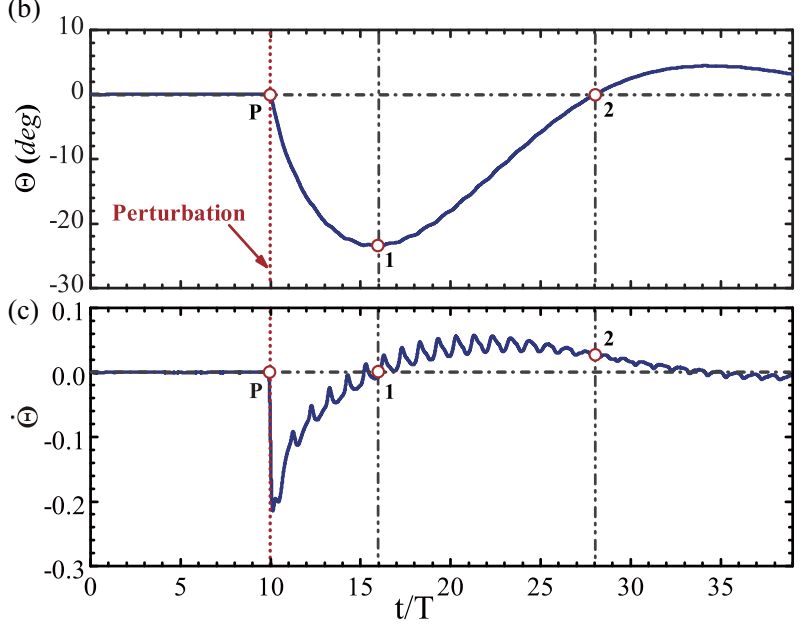

FIG. 8. Response of a flyer at the ascending state under the free-flying condition to the application of a physical perturbation: (a) trajectory of the center of gravity, (b) time history of the tilting angle, and (c) time history of the tilting angular velocity. The change of color on the trajectory from light to dark represents the increase of time. The arrows on the trajectory represent the orientations of the flyer. The control parameters used are the same as those for Fig. 5(a). The physical perturbation applied is a Gaussian pulse of torque in the form of $T_{p}=-A_{t} \exp \left[-\left(t-t_{c}\right)^{2} / 2 \tau_{c}{ }^{2}\right]$, where $A_{t}=1.0, t_{c}=10.0$, and $\tau_{c}=0.05$. Movie S5, which shows the animation of vortex structure evolution in the wake, is available as Supplemental Material [27].

design is not desirable since it can easily lead to flipping over (postural instability). However, for an object which hovers passively in oscillating flows, it has been found that the top-heavy arrangement is intrinsically stable [15]. In the computational model of this paper, the center of gravity always coincides with the geometric center. This is because the mass of the load is lumped into the mass of the flapping foils. We study the postural stability of the flyer at different locomotion states by examining its response to physical and numerical perturbations. The ascending and descending states are found to be very robust against numerical perturbations. Thus, for the ascending and descending states, we only focus on the flyer's response to physical perturbation.

Figure 8 shows the response of an ascending flyer to a Gaussian pulse of torque. It can be seen that after being perturbed at $t=10 T$, the tilting angle increases sharply and reaches its maximal value of $24^{\circ}$ after an elapse of six flapping cycles. The tilting angle then gradually diminishes and approaches zero after an elapse of another 12 flapping cycles. At this moment, the lateral displacement reaches its maximal value of roughly four body lengths. Although small fluctuations in the tilting angle and the tilting angular velocity are still observed afterward, it is reasonable to believe that the steadily ascending state can eventually be recovered. It is also worth pointing out that the alignment of the body orientation with the velocity vector (or the tangential vector along the trajectory) is clearly revealed in Fig. 8. This "weathercocking" phenomenon has previously been observed in top-heavy and bottom-heavy jellyfishlike flyers during ascending [22].

Figure 9 shows the response of a descending flyer to a Gaussian pulse of torque. The descending flyer's response is more complex than that of the ascending one and the descending state seems to be irrecoverable after being perturbed at $t=5 T$. From the zigzag trajectory, we can see that the flyer has experienced three sharp turns. During the first two turns, the flyer flips over by tilting more than $150^{\circ}$ in the clockwise and counterclockwise directions, respectively. At the end of the simulation when the flyer just finishes its third turn, the upright orientation is recovered. However, since a significant negative angular velocity still exists at this time instant, the flyer may flip over again if the simulation continues for an extra time. This motion of the flyer resembles the mixed fluttering and tumbling motion that was previously observed in falling cards [28]. 
(a)

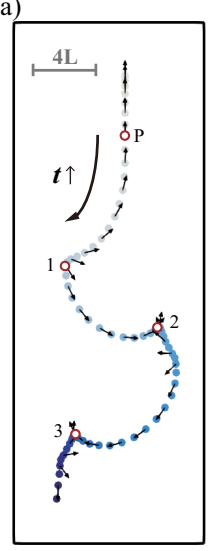

(b)

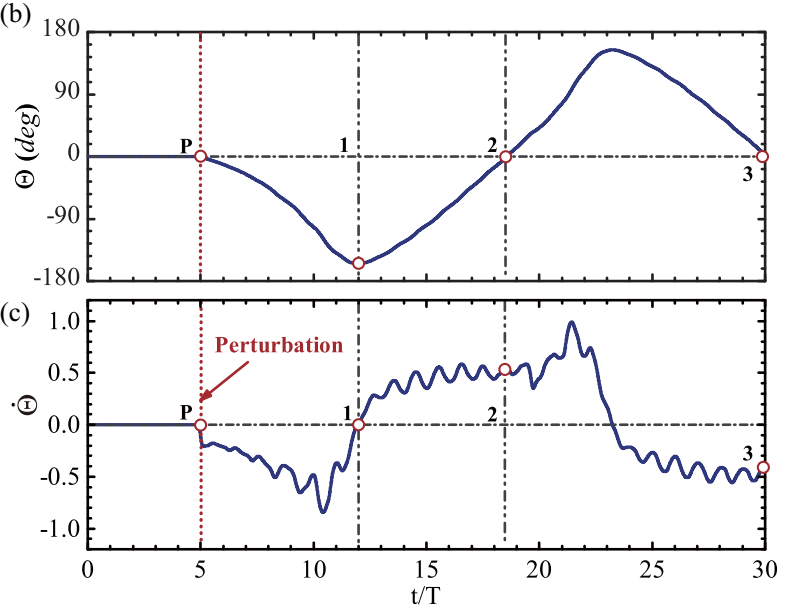

FIG. 9. Response of a flyer at the descending state under the free-flying condition to the application of a physical perturbation: (a) trajectory of the center of gravity, (b) time history of the tilting angle, and (c) time history of the tilting angular velocity. The change of color on the trajectory from light to dark represents the increase of time. The arrows on the trajectory represent the orientations of the flyer. The control parameters used are the same as those for Fig. 5(b). The physical perturbation applied is a Gaussian pulse of torque in the form of $T_{p}=-A_{t} \exp \left[-\left(t-t_{c}\right)^{2} / 2 \tau_{c}{ }^{2}\right]$, where $A_{t}=1.0, t_{c}=5.0$, and $\tau_{c}=0.05$. Movie S6, which shows the animation of vortex structure evolution in the wake, is available as Supplemental Material [27].

Figure 10 shows the responses of a flyer at the approximate hovering state to a Gaussian pulse of torque and to the numerical perturbation. From this figure we can see that both the applied physical perturbation [Fig. 10(a)] and the numerical perturbation [Fig. 10(b)] can result in the breakdown of the approximate hovering state. In both cases, the flyer experiences several sharp turns before the upright orientation is finally restored. Although the postural stability is preserved after the perturbation, the approximate hovering state is still irrecoverable in the sense that the locomotion state finally transits from hovering into ascending. Interestingly, after examining several cases of approximate hovering, with either a slightly positive or a slightly negative $U_{T}$, the only outcome after the perturbations is the transition from hovering to ascending (the transition from hovering to descending is never observed). In the absence of physical perturbation, the spontaneous occurrence of wake symmetry breaking can be regarded as the first stage during such a transition. When comparing with the constrained-flying cases, we find that the lateral forces developed under the free-flying condition are much smaller in magnitude. This can be explained by the fact that the free-flying condition actually allows the evasion of the body-vortex collision and also mitigates the excessive accumulation of vorticity near the body.

Figure 11 shows the time histories of the vertical velocities for the two cases of Fig. 10. From this figure it can be seen that after the upright posture of the flyer is almost restored $(t>55 T)$, the ascending speeds for both cases are approximately 0.6. Intriguingly, this ascending speed can also be achieved if the flapping frequency for the cases of Fig. 10 is slightly increased (by less than $10 \%)$. It is thus suggested that an ascending state with relatively small terminal speed can possibly be achieved with different sets of kinematics.

Figure 12 shows the instantaneous vorticity fields corresponding to the free-flying cases after physical perturbations. For the ascending case [Fig. 12(a)], the vorticity field looks very similar to the steadily ascending case under the constrained-flying condition [Fig. 5(a)], despite the vortices being arranged along a curved path. For the descending case [Fig. 12(b)], the vorticity field is dissimilar to that of the steadily descending case under constrained-flying condition [Fig. 5(b)]. The vorticity field actually resembles that produced by two falling cards [28]. For the approximate hovering case [Figs. 12(c)-12(e)], initially the vorticity field is the same as that under the 
(a)

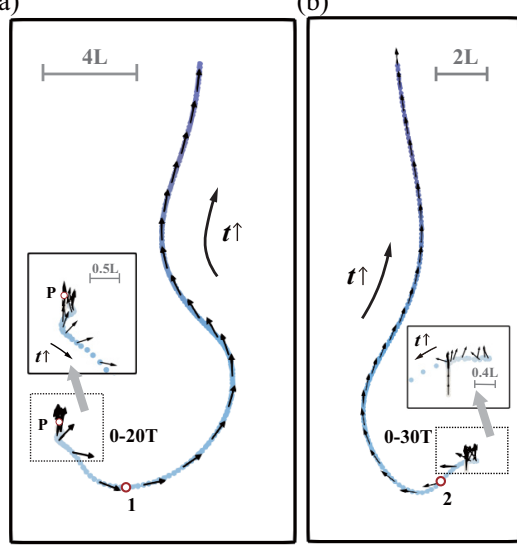

(c)

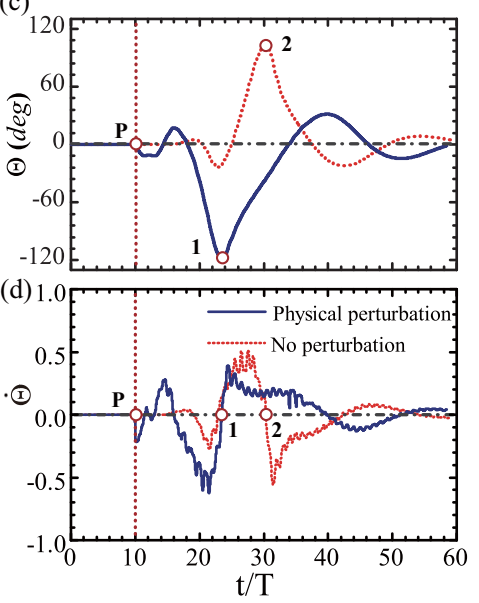

FIG. 10. Responses of a flyer at the approximate hovering state under the free-flying condition to physical and numerical perturbations: (a) trajectory of the center of gravity with a physical perturbation applied, (b) trajectory of the center of gravity without the physical perturbation, (c) time histories of the tilting angles, and (d) time histories of the tilting angular velocities. The change of color on the trajectory from light to dark represents the increase of time. The arrows on the trajectory represent the orientations of the flyer. The control parameters used are the same as those for Fig. 5(c). The enlargements of the early-time portions of the trajectories are shown in the insets of (a) and (b). The physical perturbation applied is a Gaussian pulse of torque in the form of $T_{p}=-A_{t} \exp \left[-\left(t-t_{c}\right)^{2} / 2 \tau_{c}{ }^{2}\right]$, where $A_{t}=1.0, t_{c}=10.0$, and $\tau_{c}=0.05$. In the absence of physical perturbation, the transition from the approximate hovering state to the ascending state is triggered by the numerical perturbation. Movies S7 and S8, which show the animations of vortex structure evolution in the wakes corresponding to (a) and (b), respectively, are available as Supplemental Material [27].

constrained-flying condition. When the symmetry breaking sets in, the vorticity field first looks like those shown in Fig. 6. After experiencing a few sharp turns (when the upright orientation is restored), the vorticity field becomes similar to the one shown in Fig. 12(a).

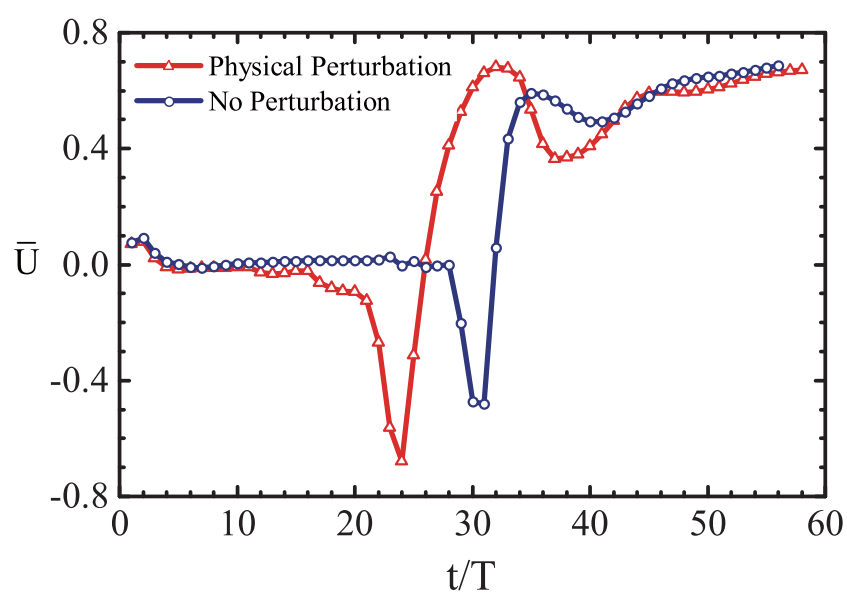

FIG. 11. Time histories of the average vertical velocity over one flapping cycle, for the two approximate hovering cases under the free-flying condition with and without the physical perturbation [corresponding to Figs. 10(a) and 10(b)]. 


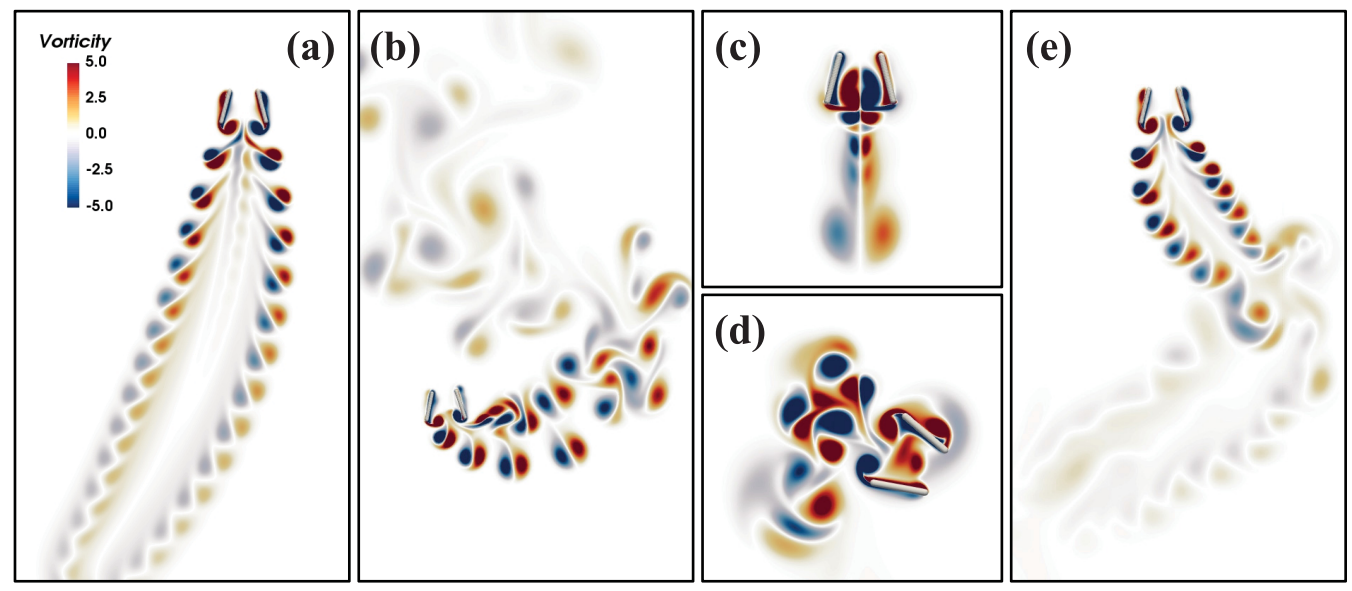

FIG. 12. Instantaneous vorticity contours for the free-flying cases after (numerical or physical) perturbations: (a) the ascending case of Fig. 8 at $t=28 T$, (b) the descending case of Fig. 9 at $t=30 T$, and (c)-(e) the approximate hovering case of Fig. 10(a) at (c) $t=10 T$, (d) $t=24 T$, and (e) $t=46 T$.

\section{CONCLUSIONS}

We numerically investigated the locomotion of a two-dimensional jellyfishlike flyer by solving the Navier-Stokes equations together with the dynamics equations. We studied the influences of some control parameters on the locomotion performance and examined the wake structures associated with different locomotion states. The symmetry-breaking instability of the wake and the postural stability of the flyer were also explored. These results provide us with some useful insights into the future design of flapping-based micro air vehicles.

A systematic parametric study has been conducted under the constrained-flying condition where the flyer is only allowed to move in the vertical direction. The dependence of the weight-supporting capacity on some control parameters was studied. With the variations in parameter values, three different locomotion states, namely, ascending, descending, and approximate hovering, were identified. The wake structures for the ascending and descending states are characterized by two columns of thrust-producing dipoles and two parallel drag-producing von Kármán vortex streets, respectively. The wake structure corresponding to the approximate hovering state is characterized by the large chunks of vorticity which linger near the flyer. The wake for the approximate hovering state was found to be susceptible to the symmetry-breaking instability. The occurrence of wake symmetry breaking can be attributed to the vorticity accumulation near the flyer due to the lack of a translation velocity. We notice that an asymmetric wake was also reported in the swimming of a two-dimensional jellyfish model when the Reynolds number exceeded 1000 [19]. Obviously, the physical mechanisms behind these two types of wake symmetry breaking are not the same.

We also conducted several case studies to explore the postural stability of a flyer, with its center of gravity located at the geometric center. The flyer's responses to physical and numerical perturbations were examined under the free-flying condition. The ascending state was found to be recoverable after the application of a strong physical perturbation, although a large lateral displacement arises. The descending state of the flyer is irrecoverable and it transits into a mixed fluttering and tumbling motion which resembles that observed previously in falling cards. The approximate hovering state is also irrecoverable, with or without a physical perturbation. For all cases studied in this work, the approximate hovering state eventually transits into the ascending state with a relatively small terminal speed. In the absence of physical perturbation, the spontaneous occurrence of the wake symmetry breaking can be regarded as the first stage during such a transition.

The wake structures that emerge in this study have some similarities to those obtained by using the inviscid vortex-sheet method [22]. The flyer's capability of preserving postural stability during 
ascending and hovering is also consistent with the observations in [22]. An interesting finding in this study is that symmetry breaking can spontaneously occur in the wake of a hovering flyer after a long-time simulation, under constrained-flying or free-flying conditions. Due to the limitation of the numerical method, the inviscid simulation in [22] is not capable of predicting the long-time behavior of the wake. The finding of the present study regarding the wake symmetry breaking remains to be experimentally confirmed, using the flying tests together with the flow visualization technique.

The jellyfishlike flapping strategy can produce a vertical force which is comparable to those produced by the insectlike flapping strokes. If the self-righting capability for maintaining postural stability is also taken into account, the jellyfishlike flapping strategy is promising in the future design of ornithopters. However, the irrecoverability of the approximate hovering state after perturbations can be a potential pitfall. Perhaps this also explains why such a strategy has not been adopted by insects to perform hovering flights. For a jellyfishlike flyer to maintain a stable hovering position for a long time, some active control measures, such as the adjustments of flapping frequency and amplitude, have to be taken.

One apparent difference exists between the hovering stability of the two-dimensional flyer studied here and that of the experimental device in [13]. In the experiment of [13], the device can wander around in a horizontal plane and keep a relatively constant height. We believe that this apparent difference can probably be attributed to the three-dimensional effect, i.e., the device in the experiment is able to evade the vortices generated during hovering while the hovering two-dimensional flyer is unavoidably subjected to strong vortex-body interactions.

There are several avenues for future research. The influence of the location of the center of gravity (such as the bottom-heavy or top-heavy designs) on the postural stability remains unexplored. The power consumption and efficiency and the effect of passive flexibility on hovering performance also need to be investigated. To aid the practical design, the weight-supporting capability and postural stability of a more realistic three-dimensional model should be assessed.

\section{ACKNOWLEDGMENTS}

This work was supported by Chinese Academy of Sciences under Projects No. XDB22040104 and No. QYZDJ-SSW-SYS002; National Natural Science Foundation of China under Projects No. 11772338, No. 11372331, No. 11232011, and No. 11572331; and Ministry of Science and Technology of China under the 973 Program No. 2013CB834100. We would like to thank the National Supercomputing Center in Tianjin for the allocation of computing time.

\section{APPENDIX A: CODE VALIDATION}

To check the accuracy of the Navier-Stokes solver in handling moving boundary problems, we simulate the flow around one cross section of a dragonfly wing during hovering flights. The idealized kinematics shown in Fig. 13 can be prescribed as

$$
\begin{aligned}
{[x(t), y(t)] } & =\frac{A_{0}}{2}[1-\cos (2 \pi f t)][1,0], \\
\alpha(t) & =\frac{\pi}{2}-B \sin (2 \pi f t) .
\end{aligned}
$$

Here $[x(t), y(t)]$ is the location of the center of the chord, $\alpha(t)$ is the orientation angle of the wing with respect to the $x^{+}$direction, $f$ is the frequency, and $A_{0}$ and $B$ are the amplitudes of the translation and rotation, respectively. The control parameters in the simulation are $f=1, B=\pi / 4$, and $A_{0}=2.5 c$. The Reynolds number based on the maximum velocity of the wing is defined as $\operatorname{Re}_{m}=U_{\max } c / v=$ $\pi A_{0} f c / v$. We compare our result at $\operatorname{Re}_{m}=150$ with the existing numerical data in Refs. [1,25]. The time histories of the vertical force coefficient $C_{V}$ and the horizontal force coefficient $C_{H}$ are shown in Fig. 14. 

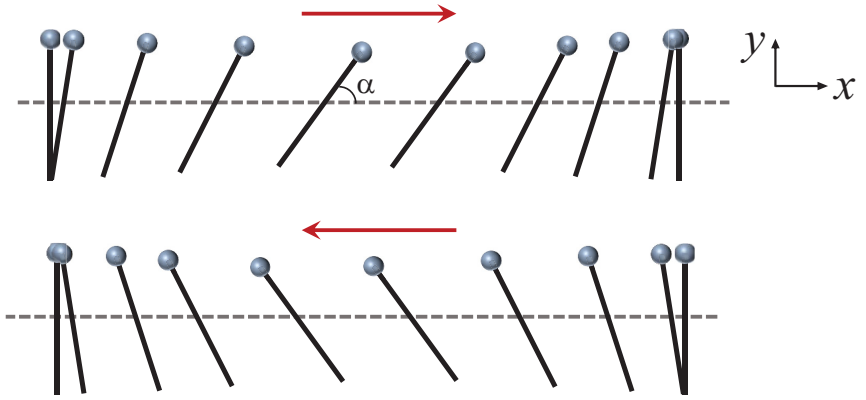

FIG. 13. Schematic diagram for the idealized kinematics of one cross section of a dragonfly's wing. The solid line represents the cross section and the closed circle represents the leading edge. The arrow denotes the direction of the translational motion during each half stroke.

Overall, the result of the present simulation agrees well with previous numerical studies, except for some differences near the crests and troughs when compared with Ref. [25] and a phase lag when compared with Ref. [1]. These discrepancies are attributed to the differences in the numerical models. The numerical model considered here is a thin rigid plate, whereas the model in [25] is a rigid ellipse and the model in [1] is a thin flexible plate.

\section{APPENDIX B: GRID AND TIME-STEP CONVERGENCE STUDY}

To test the convergence of the solutions to the refinement of grids, two cases corresponding to the ascending and descending states are simulated by using three effective mesh resolutions, where the finest grids near the flyer are $0.04 L, 0.02 L$ and $0.01 L$ in spacing, respectively. The control parameters for the ascending case are $\operatorname{Re}=300, \mathrm{Fr}=2.89, \beta=0.5, A_{\theta}=\pi / 12, \theta_{0}=0^{\circ}, d=1.0 L$,
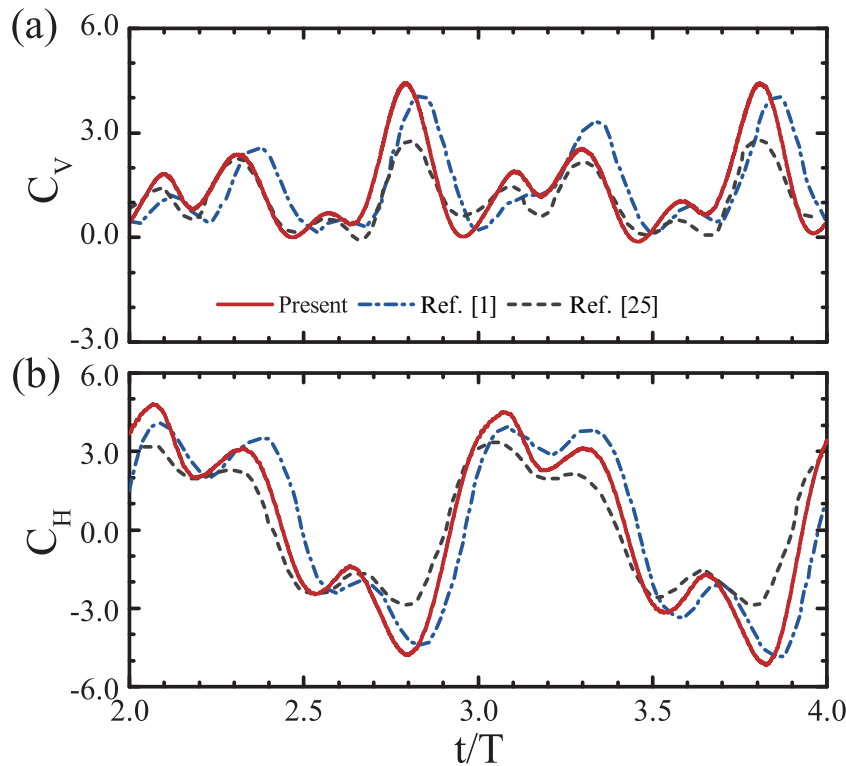

FIG. 14. Comparisons of the present result with the data from previous numerical simulations: (a) time histories of the vertical force coefficient $C_{V}$ and (b) time histories of the horizontal force coefficient $C_{H}$. 
(a)
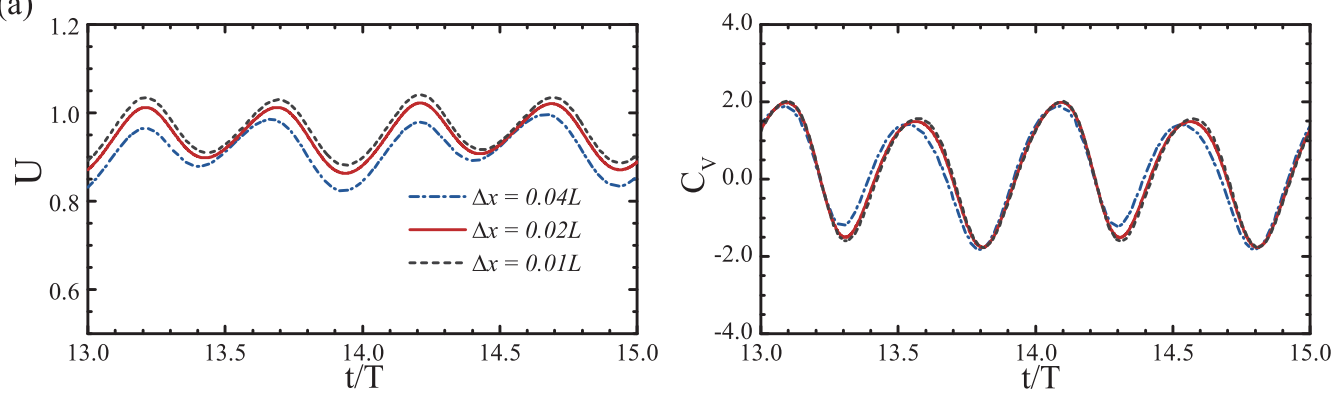

(b)
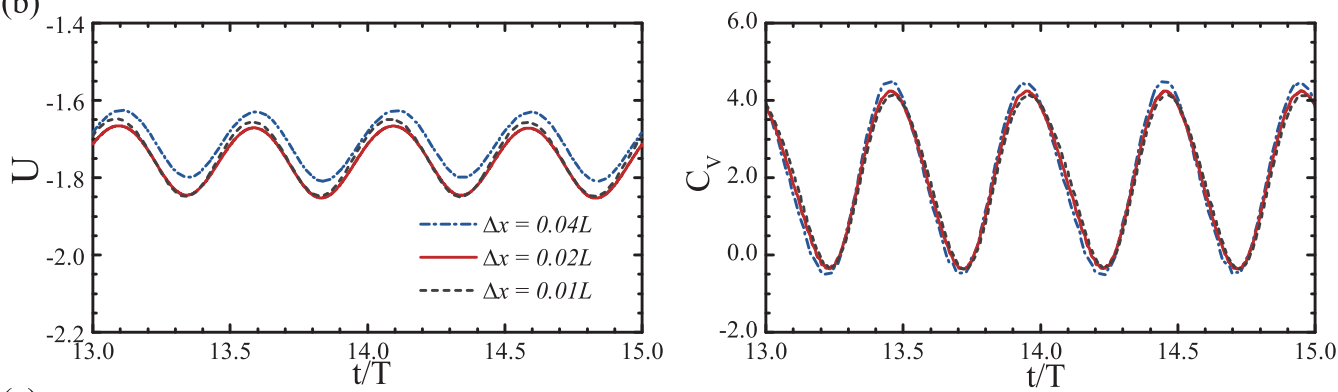

(c)
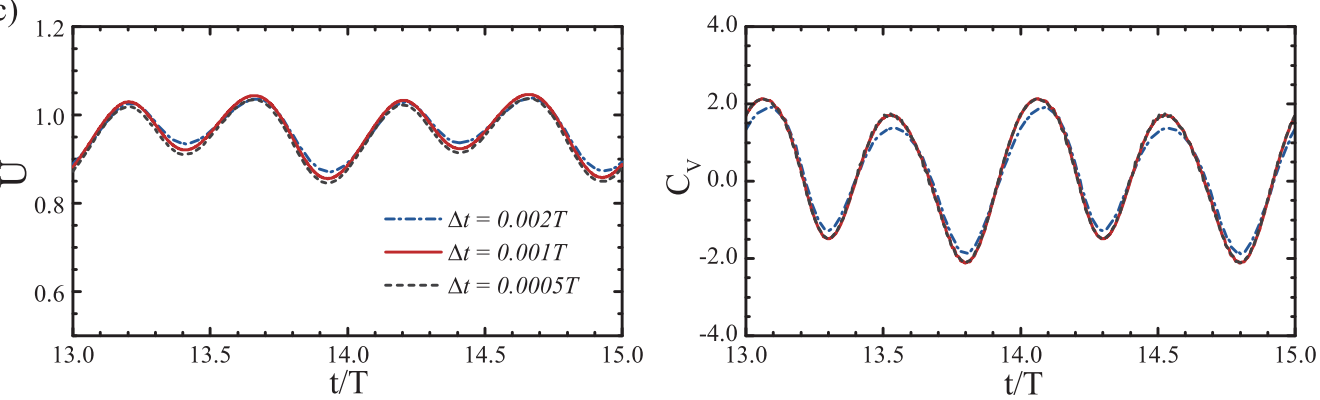

FIG. 15. Grid and time-step convergence behaviors in the time histories of the vertical speed $U$ and the vertical force coefficient $C_{V}$ : (a) grid convergence behaviors for the ascending state, (b) grid convergence behaviors for the descending state, and (c) time-step convergence behaviors for the ascending state.

and $L_{0}=0.2 L$. For the descending case, the parameters used are $\operatorname{Re}=100, \operatorname{Fr}=0.96, \beta=0.5$, $A_{\theta}=\pi / 12, \theta_{0}=0^{\circ}, d=1.0 L$, and $L_{0}=0.2 L$.

Figures 15(a) and 15(b) show the time histories of the vertical force coefficient $C_{V}$ and the vertical speed $U$ which are obtained at different mesh resolutions and the grid convergence behaviors can be clearly seen from this figure. Since the difference between the solutions obtained with $\Delta x=0.02 L$ and $\Delta x=0.01 L$ is sufficiently small, the grid width of $\Delta x=0.02 L$ is used in the simulations. For cases with higher Re numbers, finer grids are used and similar tests are also conducted to ensure the grid convergence of the solutions.

In the tests above, the time step is fixed to $\Delta t=0.001 T$. Here simulations are also conducted to test the effect of the time-step size on the solution. Figure 15(c) shows the time histories of the vertical force coefficient $C_{V}$ and the vertical speed $U$ for the ascending case, which are obtained at three time-step sizes of $0.002 T, 0.001 T$, and $0.0005 T$ (the grid spacing is fixed to $\Delta x=0.02 L$ ). It can be seen that $\Delta t=0.001 T$ is sufficient to obtain a time-step-independent solution. In the simulations of this study, $\Delta t=0.001 T$ is used for the ascending and descending cases. Since the stability property of the approximate hovering cases is very sensitive to the numerical settings, $\Delta t=0.0005 T$ is used to capture the dynamics more precisely. 
[1] S. K. Jones, R. Laurenza, T. L. Hedrick, B. E. Griffith, and L. A. Miller, Lift vs. drag based mechanisms for vertical force production in the smallest flying insects, J. Theor. Biol. 384, 105 (2015).

[2] C. P. Ellington, C. van den Berg, A. P. Willmott, and A. L. R. Thomas, Leading-edge vortices in insect flight, Nature (London) 384, 626 (1996).

[3] M. H. Dickinson, F. O. Lehmann, and S. P. Sane, Wing rotation and the aerodynamic basis of insect flight, Science 284, 1954 (1999).

[4] M. Sun and J. Tang, Unsteady aerodynamic force generation by a model fruit fly wing in flapping motion, J. Exp. Biol. 205, 55 (2002).

[5] T. Weis-Fogh, Quick estimates of flight fitness in hovering animals, including novel mechanisms for lift production, J. Exp. Biol. 59, 169 (1973).

[6] P. Zdunich, D. Bilyk, M. MacMaster, D. Loewen, J. De Laurier, R. Kornbluh, T. Low, S. Stanford, and D. Holeman, Development and testing of the mentor flapping-wing micro air vehicle, J. Aircraft 44, 1701 (2007).

[7] F. Breugel, W. Regan, and H. Lipson, From insects to machines: A passively stable, untethered flappinghovering micro air vehicle, IEEE Robot. Autom. Mag. 15, 68 (2008).

[8] G. De Croon, K. De Clercq, R. Ruijsink, B. Remes, and C. de Wagter, Design, aerodynamics, and visionbased control of the delfly, Int. J. Micro Air Veh. 1, 71 (2009).

[9] C. T. Bolsman, J.F. L. Goosen, and F. Van Keulen, Design overview of a resonant wing actuation mechanism for application in flapping wing MAVs, Int. J. Micro Air Veh. 1, 263 (2009).

[10] C. Richter and H. Lipson, Untethered hovering flapping flight of a 3D-printed mechanical insect, Artif. Life 17, 73 (2011).

[11] L. Hines, D. Campolo, and M. Sitti, Liftoff of a motor-driven, flapping-wing microaerial vehicle capable of resonance, IEEE T. Robot. 30, 220 (2014).

[12] Q.-V. Nguyen, W. L. Chan, and M. Debiasi, in Bioinspiration, Biomimetics, and Bioreplication 2015, edited by A. Lakhtakia, M. Knez, and R. J. Martín-Palma, SPIE Proc. Vol. 9429 (SPIE, Bellingham, 2015), p. 94290 U.

[13] L. Ristroph and S. Childress, Stable hovering of a jellyfish-like flying machine, J. R. Soc. Interface 11, 20130992 (2014).

[14] S. Childress, N. Vandenberghe, and J. Zhang, Hovering of a passive body in an oscillating airflow, Phys. Fluids 18, 117103 (2006).

[15] B. Liu, L. Ristroph, A. Weathers, S. Childress, and J. Zhang, Intrinsic Stability of a Body Hovering in an Oscillating Airflow, Phys. Rev. Lett. 108, 068103 (2012).

[16] Y. Y. Huang, M. Nitsche, and E. Kanso, Stability versus maneuverability in hovering flight, Phys. Fluids 27, 061706 (2015).

[17] X. M. Shao, X. L. Zhang, Z. S. Yu, and J. Z. Lin, Numerical studies on the dynamics of an open triangle in a vertically oscillatory flow, J. Fluid Mech. 788, 381 (2016).

[18] Y. Y. Huang, M. Nitsche, and E. Kanso, Hovering in oscillatory flows, J. Fluid Mech. 804, 531 (2016).

[19] S. Alben, L. A. Miller, and J. F. Peng, Efficient kinematics for jet-propelled swimming, J. Fluid Mech. 733, 100 (2013).

[20] V. Raspa, R. Godoy-Diana, and B. Thiria, Topology-induced effect in biomimetic propulsive wakes, J. Fluid Mech. 729, 377 (2013).

[21] P. A. Dewey, D. B. Quinn, B. M. Boschitsch, and A. J. Smits, Propulsive performance of unsteady tandem hydrofoils in a side-by-side configuration, Phys. Fluids 26, 041903 (2014).

[22] F. Fang, K. L. Ho, L. Ristroph, and M. J. Shelley, A computational model of the flight dynamics and aerodynamics of a jellyfish-like flying machine, J. Fluid Mech. 819, 621 (2017).

[23] S. Z. Wang and X. Zhang, An immersed boundary method based on discrete stream function formulation for two-and three-dimensional incompressible flows, J. Comput. Phys. 230, 3479 (2011).

[24] S. Z. Wang, G. W. He, and X. Zhang, Parallel computing strategy for a flow solver based on immersed boundary method and discrete stream-function formulation, Comput. Fluids 88, 210 (2013).

[25] Z. J. Wang, The role of drag in insect hovering, J. Exp. Biol. 207, 4147 (2004).

[26] D. B. Quinn, K. W. Moored, and A. J. Smits, Unsteady propulsion near a solid boundary, J. Fluid Mech. 742, 152 (2014). 
[27] See Supplemental Material at http://link.aps.org/supplemental/10.1103/PhysRevFluids.3.013102 for the animations of vortex structure evolution in the wakes.

[28] A. Andersen, U. Pesavento, and Z. J. Wang, Unsteady aerodynamics of fluttering and tumbling plates, J. Fluid Mech. 541, 65 (2005). 